\title{
Harmless? Mixed perception and awareness of vitamin and mineral supplements
}

\author{
V. Marinello, C. Buckton and E. Combet \\ Human Nutrition, School of Medicine, College of MVLS, University of Glasgow, Glasgow, Scotland, G12 $8 Q Q$
}

Vitamin and mineral supplements (VMS) have seen an increase in consumption (40\% to over $50 \%$ of US adults in 1988 compared to $2006)$ in recent years ${ }^{(1)}$, coinciding with an increasing public focus on health improvement. Large scale epidemiological research suggests VMS offer no health benefit to the general healthy population, and more recently evidence has shown supplement use can increase risk of mortality. ${ }^{(2)(3)}$ This study used semi-quantitative and qualitative techniques through a word-association projective exercise and focus groups to explore public perceptions of VMS. The four prompt terms were "Antioxidants", "Dietary Supplements", "Vitamins" and a picture prompt depicting various VMS bottles. FG used a topic guide centred around key research questions to explore public perceptions of VMS.

Results from 427 participants were obtained (27\% supplement users), with a majority of responses elicited being blank or "I don't know" (45\%). The next most popular responses indicated that VMS are most strongly associated with the themes of "health", "vitamin \& mineral types", "food types" and "formulation". More females than males associated VMS with being "bad for you", and males associated VMS with the "medical" theme more than females. Younger participants (<45years) gave more responses than older participants under the themes "fake/cheat", "natural" and "blank/no idea" with older participants more likely to associate VMS with "deficiency/disease/specific need". Those from more deprived areas had higher responses associated with the theme "good for you" than those from less deprived areas. Differences in responses were also found according to the prompt terms used. Both "Vitamins" and the picture prompt had stronger association with "Health in General" $(\mathrm{p}<0.001)$ than other prompts and "Vitamins" had less responses reflective of the theme "bad for you" than all other prompts $(\mathrm{p}=0 \cdot 034)$. "Antioxidants" had more "blank" responses $(53.7 \%, \mathrm{p}<0.001)$ and responses in the themes "science talk" $(\mathrm{p}<0.001 ; 2 \cdot 2 \%)$, "cleansing/detox" ( $\mathrm{p}<0.001$; $3.5 \%)$ and "medical" ( $<<0.001 ; 3.1 \%)$ than other prompts. Dietary Supplements elicited responses more reflective of the industry with "Weight Loss" and "Shops \& Brands" within the top five themes.

Five FG were carried out ( $\mathrm{n}=19 ; 68 \%$ female; median age 30; $68 \%$ supplement users). The focus groups revealed mixed opinions about VMS, which were generally perceived to be unnecessary with vitamins and minerals from food being seen as the best option. However in the advent of illness, a specific need or more worryingly an insufficient diet VMS were perceived to be of benefit. VMS were not considered to be harmful and information from public health bodies or experts about adverse effects was unlikely to influence participants' decision to use them. This coupled with the fact they would not be influenced by evidence regarding their safety has important public health implications, as this is indicative of the lack of trust the public have in public health nutrition messaging.

1. Gahche J, Bailey R, Burt V, Hughes J, Yetley E, Dwyer J, Picciano MF, McDowell M, Sempos C. (2011). NCHS data brief. 611-8.

2. Bjelakovic G, Nikolova D, Gluud LL, Simonetti RG, Gluud C. (2007). Jama. 8, 842-57.

3. Myung SK, Kim Y, Ju W, Choi HJ, Bae WK. (2010). Annals of Oncology. 1, 166-79. 